\title{
Transformación digital en la educación del Perú, en tiempos de pandemia
}

Dra. Lizaura Arribasplata Rodas

Lizaura14@gmail.com Universidad César Vallejo

Lima-Perú

Mag. Nelly Luisa de Gonzaga Zarzosa de Rivera

Zarzosan_21@yahoo.es

Universidad César Vallejo

Lima-Perú

\section{RESUMEN}

La presente investigación tuvo como objetivo resaltar la importancia y los beneficios que obtienen las escuelas, instituciones y universidades tras a la implementación de la tecnología, así como demostrar que debido a la coyuntura actual es indispensable que en el sector educación los docentes se encuentren dispuestos a trabajar con dicha implementación, esto con el propósito de lograr atribuirles una enseñanza adecuada a sus alumnos. La metodología utilizada dentro del presente artículo ha sido bajo un enfoque cuantitativo, por medio del análisis de diversos datos bibliográficos de organismos de nivel nacional como también de algunos autores con nociones que guardan concordancia con el tema tratado. Finalmente, se concluyó que debido al COVID-19, el sector educación ha tenido que adaptarse a las nuevas circunstancias. Por ende, el Estado peruano ha tenido que brindar las facilidades para que dicho sector comience a emplear la tecnología, la cual ha ayudado a que tanto docentes como estudiantes puedan seguir desarrollando sus actividades con normalidad.

Palabras clave: tecnología digital; educación; pandemia. 


\title{
Digital transformation in education in Peru, in times of pandemic
}

\begin{abstract}
The present research aimed to highlight the importance and benefits that schools, institutions and universities obtain after the implementation of technology, as well as to demonstrate that due to the current situation it is essential that in the education sector teachers are willing to work with this implementation, this with the purpose of achieving attributing adequate teaching to their students. The methodology used in this article has been under a quantitative approach, through the analysis of various bibliographic data from national organizations as well as from some authors with notions that are consistent with the subject matter. Finally, it was concluded that due to COVID-19, the education sector has had to adapt to the new circumstances. Therefore, the Peruvian State has had to provide the facilities for said sector to start using technology, which has helped both teachers and students to continue developing their activities normally.
\end{abstract}

Keywords: digital technology; education; pandemic.

Artículo recibido: 15 noviembre. 2021 Aceptado para publicación: 10 diciembre 2021

Correspondencia: Lizaura14@gmail.com Conflictos de Interés: Ninguna que declarar 


\section{INTRODUCCIÓN}

En la actualidad se logra apreciar que el mundo está pasando por problemas sanitarios, esto llevando a que los diversos países tomen conciencia sobre la proliferación e incremento masivo que tiene el poder contagiarse en las personas. Siendo así que se han vivido una de las situaciones más críticas en la historia de la humanidad a consecuencia de la pandemia del Covid-19, uno de los cambios fue el aislamiento social, nuevas medidas de actividades y distanciamiento social, en prácticamente todas las naciones lo cual ha afectado severamente la vida cotidiana y las acciones de todos los ciudadanos. Es importante mencionar que los sectores de educación, salud y otros tuvieron muchos cambios por ejemplo la educación a nivel mundial tuvo un impacto muy significativo afectando tanto a los estudiantes, docentes, personal administrativo y autoridades. Del mismo modo afecto a otros sectores relacionados con la educación en colegios, universidades o institutos superiores por lo que han tenido que implementar nuevas estrategias y creatividad para enfrentar los problemas a consecuencia de la crisis del Covid-19 y se implementó en diversos centros de estudios los medios digitales como un método de enseñanza y aprendizaje (Ordorika, 2020). Del mismo modo el Ministerio de Educación tomo como salida la implementación de herramientas tecnológicas a distancia, siendo así que estos emplearon páginas webs, uso de aplicaciones móviles para el contacto interno con los estudiantes, además de diversas plataformas para poder realizar el desarrollo de estas, siendo así que las instituciones tuvieron que buscar de estrategias las cuales sean más efectivas para que empleen un desarrollo adecuado en las labores estudiantes, así como se realiza de manera presencial.

Asimismo, se puede definir a la tecnología educativa que se implementó recientemente de manera masiva por los problemas que se observaron en el mundo con la pandemia. Siendo así que la tecnología y los dispositivos digitales permiten interactuar y establecer conexión con diversos ordenadores y otros dispositivos en el mundo, lo cual en el XXI se observó el uso a menudo ya que estos requerían de interactuar y comunicarse como si se estuviera realizándose manera presencial; pero estos campos no se desarrollando ampliamente en el área de educación. Sin embargo, se reconoció que hubo un gran avance en toda América Latina en poder implementar tecnología ante la coyuntura por la que se está atravesando, observándose como derecho el poder brindar y tener una correcta educación de calidad; pero aún queda mayores áreas en donde se deba mejorar el aspecto 
del desarrollo de los recursos tecnológicos que tiene cada país. También se observa como un punto en contra del desarrollo óptimo de la educación para los niños, que es la capacidad que tiene cada estado en poder brindar un correcto presupuesto para invertir en el desarrollo de las instalaciones educativas y de los conocimientos de los propios maestros, ya que así estos podrán brindar un correcto nivel de educación para los niños (Cueva,2020).

Por otro lado, reconociendo que la pandemia del Covid-19 en donde se paralizo todas las actividades en espacios cerrados, en espacios abiertos y respetar el aforo indicado por las autoridades respectivas, este no fue el caso de la educación ya que en ello se empleó diversas herramientas tecnológicas en donde se buscó seguir con las clases de los estudiantes, siendo así que se busca el reemplazo de escena; ya que debido a la pandemia se llevó a transformar las clases presenciales a clases virtuales, permitiendo que los estudiantes no se contagien y contagien a sus familiares con el virus. Pero también se tuvo que reconocer como se encontraban las instituciones educativas con respecto a la realidad que pasaban estos, ya que muchas escuelas se observan en deterioro o no cuentan con la suficiente capacidad de recursos para poder brindar las herramientas a los docentes, permitiendo así que estos puedan ejecutar clases activas y benéficas para los estudiantes. Además, en donde los maestros deben tener conocimiento en el implementar y usar las herramientas tecnológicas que se emplea para poder brindar clases virtuales de una manera óptima a fin de la compresión de todos los estudiantes (Aguilar, 2020).

Del mismo modo, se menciona que debido a la pandemia y la sociedad del conocimento al implementar el uso de las tecnologias de informaciòn y comunicaciòn en el aprendizaje de cada estudiante en el aprendizaje virtual. En el Perù la realidad social vinculante a la educacion no ha sido la màs optima ya que muchos estudiantes no cuentan con los recursos necesarios para llevar una educaciòn a distancia,por lo que es necesario que se implementen nuevas estrategias en la educaciòn para el beneficio de cada uno de los estudiantes (Huanca et al.,2020).

También se evidencia que los docentes pasaron con un cambio radical de los sistemas que empleaban con normalidad en las clases antes de la pandemia del COVID-19, ya que el Ministerio de Educación velo por la seguridad de los propios maestros y de los estudiantes, en donde se observó que estos tuvieron que acoplarse a las diversas necesidades que se tenían en la institución o los escasos recursos que brindaba el Estado 
con respecto a las herramientas que se debe tener para poder continuar con las clases de los estudiantes. Pero también existieron algunos problemas/imprevistos que escapaban de las manos de los propios docentes o alumnos, ya que se reportaban casos de falla de conexión, bajos rangos de cobertura, la conectividad, etc. Siendo estos algunos problemas por los cuales los niños y maestros pasaban para poder continuar con las clases, además se observaron que en diversos casos los maestros no reconocen de manera adecuada la adopción de las tecnologías para el proceso educativo; lo cual retrasa en el desarrollo de estos (García Lear et al., 2021).

Y finalmente los autores Cayo y Agramonte (2020) señalan que la principal causa de toda la innovacion de la tecnologia digital fue a causa de la pandemia del Covid-19 ya que se tuvo que adoptar medidas en materia de salud pùblica para evitar los contagios masivos en todo el pais y el mundo. Por lo que fue necesario paralizar o deterner de inmediata todas las actividades en diferentes colegios, universidad del mundo. Por otro lado, el desarrollo tecnològico fue crucial para poder llevar a cabo distintas actividades en los procesos educativos y se dio la migración de la modalidad presencial a una innovadora modalidad virtual.

\section{ESTRATEGIAS METODOLÓGICAS O MATERIALES Y MÉTODOS}

La presente investigación se realizó con un enfoque cuantitativo,de acuerdo con Guedes dos Santos et al. (2017) señalo que una investigación cuantitativa se basa en la elaboración de estudios que están vinculados a datos númericos, los cuales fomentan la conjunción de detalles e información de esta manera permita corroborar teorias y/o hipótesis, asimismo se emplea el análisis estadístico. En la misma linea Tobi y Kampen (2017) señalan que esta permitió evidenciar los resultados de manera numérica y cuantificar los productos en aspectos estadísticos, asimismo recopilar información a manera de verificar o corroborar las hipótesis y/o teorías planteadas.

\section{Técnicas e instrumentos de recolección de datos}

Por otro lado, los datos utilizados se han originado a partir de una relación hipotéticadeductiva, la que nos permitirá verificar la credibilidad de los mismos, siendo que la verificación será de manera empírica. Por ende, se refiere a las variables para poder llegar a una conclusión en este caso sería la hipótesis para verificar su credibilidad y veracidad, y en caso el resultado fuera optimo se concluiría que hay una relación con la teoría inicial y los planteamientos del problema de la investigación (Sánchez,2019). 
Así también, los datos utilizados se han originado a partir de una relación hipotéticadeductiva, siendo así que la verificación será de manera empírica por lo que las variables han sido estudiadas por la relación entre ellas, esto con la finalidad de obtener un mayor entendimiento y comprensión de las mismas en conjunto, así como de su importancia de investigación (Núñez,2017).

\section{Procedimiento}

Se realizó la búsqueda bibliográfica y sistemática de artículos científicos en las siguientes bases de datos: Redalyc, Scielo y Scopus. La búsqueda y selección de los artículos inició en el período 2017 hasta el año 2021, para la investigación se decidió la selección de los artículos bajo una estructura relacionada con las variables, que consistió en realizar una búsqueda de información enfocada en la trasformación digital en la educación en el Perú, sus características, normativa nacional, beneficios, también se tomó en consideración al sector educación a nivel nacional, sus características, perspectiva de los docentes, perspectiva de los alumnos, entre otros, y su relación con la implementación de la tecnología, y su relación con el aprendizaje a consecuencia de la Covid-19, enfocándonos en la incidencia de una variable sobre la otra.

Es por ello que, los datos obtenidos tienen el objetivo de ser desarrollados de manera correcta en el presente trabajo, dado que son parte de una revisión bibliográfica ardua relacionada a la temática mencionada anteriormente, los criterios para su selección fueron la fecha de publicación, el contenido veraz y verificable de la información, así es como se desarrollan; esta será utilizada como sustento estadístico en el análisis que se llevará a cabo.

\section{RESULTADOS Y DISCUSIÒN}

\section{Resultados}

Es así que, se evidenció que la transformación digital en la educación es realmente importante, dado que actualmente debido a la pandemia, todo se realiza de manera virtual haciendo empleo de las herramientas informáticas para desarrollar sus actividades fundamentales como el estudiar, trabajar, comunicarse; por lo cual, constituye un problema o barrera para los ciudadanos el que no todos puedan tener acceso a los medios digitales, para lo cual se deberían plantear estrategias por parte del gobierno, en favor de los ciudadanos y su desarrollo en la sociedad. Asimismo, se llega a considerar un reto el que las personas puedan crecer en el ámbito profesional con las posibilidades de poder 
contar con las oportunidades que el Estado puede brindar a través de sus diversos programas con el objetivo de no parar con la educación y que esta pueda llegar a todos los sectores del Perú con el fin de no dejar a ninguna persona sin la posibilidad de ayudarlos profesionalmente y durante ese proceso reducir la cantidad de casos sobre barreras tecnológicas.

De tal manera que, además de volverse necesarias, logró impactar en diferentes sectores como el sector social; lo cual permitió el que el gobierno dé luz verde a ello y apoye a los estudiantes brindándoles tablets para el desarrollo de sus clases, por lo cual, llegó a la mayoría de personas, pero no a todas; es así que se considera aún en proceso de cumplir con sus propuestas. Gajardo y Díez (2021) señalan que una de las consecuencias de la pandemia fue el sector de la educación, no solo significó el cierre de las instituciones educativas o universitarias, sino afecto el proceso de aprendizaje de todos los estudiantes por lo que se implementó diversas herramientas tecnológicas que faciliten el proceso de aprendizaje a distancia, entre ellas fueron las plataformas educativas como zoom o meet que son las más conocidas en el nivel educacional que puedan ayudar a la comunicación e interacción entre el alumno y docente. Otro factor importante relacionado a la educación fue la falta de recursos como el internet ya que esto es necesario para poder llevar clases en línea a través de las plataformas educativas y en varios países no se contaba con lo necesario para llevar clases virtuales, por lo que se tuvo que generar soluciones como adelantar el periodo de vacaciones o actividades directamente al hogar con materiales que todos tengan en casa. Debido a los factores que imposibilitan la educación a distancia se tuvo una planificación con el fin de asegurar e implementar una educación a distancia con objetivos necesarios para el aprendizaje de cada estudiante, pero en un corto tiempo se presentaron muchas dificultades como falta de internet, falta de recursos tecnológicos que conlleva una educación global a distancia ante una situación para la que no se estaba preparado. Por otro lado, los docentes se vieron afectados por esta nueva modalidad de una docencia presencial a una educación virtual, por lo que implementaron propuestas de calidad en la educación con sus propios recursos o herramientas ya que muchas veces no contaban con lo necesario ni apoyo por las autoridades, y no contaban con formación digital muy diversa y frecuentemente escasa.

Por otro lado, Connor et al. (2020) refieren que deben brindarse nuevas herramientas tecnológicas que benefician al desarrollo del aprendizaje de los estudiantes entre las 
plataformas más usadas en los últimos años son zoom, meet o plataformas del mismo centro de estudio que brinden y faciliten las clases virtuales. Por otro lado, debido a la pandemia un sector afectado son las familias ya que muchos de ellos fueron afectados por la falta de trabajo, los despidos masivos, la economía, la salud, la educación para sus hijos se vio afectada, cada uno de ellos confinados en el hogar, implementaron los recursos digitales, red de internet y espacios de teletrabajo con la obligación o seguir con normalidad sus actividades como son las actividades de aprendizaje desde casa a través de las plataformas educativas, tareas o conferencias para los estudiantes pero ha generado muchos cambios en cada uno de los alumnos y genera malestar tanto físico como emocional debido a los cambios por la pandemia de la Covid-19.

Levano et.al (2019) precisan que el campo digital se ha transformado de acuerdo a las actividades y roles de cada ser humano, como lo es el uso de internet que ha logrado revolucionar todos los campos de manera rápida y eficaz para hacer empleo de las tecnologías digitales así como de los recursos que sirven para aquellos ambientes de aprendizaje, como de complejidad y de crecientes oportunidades de acuerdo al entorno y provecho que le sepa sacar cada persona a su productividad tecnológica involucrando una serie de procesos para cumplir y desarrollar todas las competencias que este campo necesita.

Por otro lado, Argüelles et al. (2021) refieren que la eduaciòn en Amèrica Latina cambio a consecuencia de la pandemia de la Covid-19 por lo que se recurrio al uso de estrategias de comunicación a distancia ante el escenario de consecuencias sanitarias, sociales y económicas derivadas de la pandemia de la COVID-19. Esto causa que más del $70 \%$ de estudiantes se vio afectado por las medidas establecidas como el confinamiento social, aislamiento social esto también afecto drásticamente la calidad de la enseñanza y la educación en la región. En lo que se representa esta medida se desarrolló conocimientos de calidad a través de las plataformas virtuales y los esfuerzos de los docentes para profesionalizarse de una manera más innovadora frente a la enseñanza digital y remota. Y finalmente, Iivari et al. (2020) refieren que la pandemia del Covid-19 incio una trasformacion digital extensa en toda la sociedad por lo que se dio la innovacion de los recursos digitales incluidos en la educaciòn. Por otro lado se menciono en los resultados que la educación se transformó de una práctica tradicional en el aula a una remota y digitalizada. Esto requirió ajustes importantes no solo de los niños y sus maestros, sino 
también de sus familias, la administración escolar y toda la sociedad. Cabe resaltar que debido a esta implementación las brechas digitales que identificamos se muestran desafortunadamente fuertes en la nueva normalidad actual de COVID-19 porque no todos los niños están en la misma posición para participar en su educación básica digitalizada. Existen diversos problemas con el acceso y el uso de la tecnología tanto entre los adultos como entre los niños involucrados, incluso puede haber problemas con el acceso a Internet, los dispositivos y las aplicaciones necesarias porque no todos tienen la posibilidad económica para implementar esos recursos en el hogar.

\section{Discusión}

En una de sus conclusiones, Gómez \& Escobar (2021) identificaron que los ciudadanos no se encuentran en igualdad de condiciones en cuanto al acceso de las herramientas tecnológicas, en especial, para la educación virtual, dado que las brechas de desigualdad siempre han estado presentes, sin embargo, durante la pandemia fue más notorio. En el caso de las instituciones educativas, se pudo observar que el $71 \%$ que pertenecía a instituciones secundarias tenían acceso a las tecnologías de información, mientras que el $37 \%$ de instituciones tenía acceso a internet. Entre lo que respecta a las familias, el $92 \%$ de ellas contaba por lo menos con una herramienta digital, mientras que el 7\% no tenía acceso a ninguna de ellas. Asimismo, en el caso de los docentes el 20\% de los docentes rurales y un $15 \%$ de docentes de instituciones públicas no contaban con una laptop o computadora en casa. Estos datos logran evidenciar que la situación en cuanto a la transformación digital, es un proceso que se da en el Perú de manera lenta, lo cual afecta a muchos ciudadanos en su desarrollo.

De la misma manera, Anaya et.al (2021) pudieron determinar que la tras la llegada del COVID-19 se ha podido destacar la existencia de una rotura de carácter estructural, siendo que está venía desde épocas pasadas. Lográndose evidenciar aún más que existe una notable diferencia entre las áreas urbanas con las áreas rurales esto en cuanto al sector educación, puesto que se precisa que en las primeras áreas mencionadas los colegios cuentan con una infraestructura adecuada para que los alumnos puedan adquirir una educación de calidad y sobre todo en un ambiente seguro, mientras que en la zonas rurales los órganos gubernamentales tienden a tener ciertas limitaciones al momento de apoyar en la mejora de las infraestructuras de los colegios, ya que solo invierten en los implementos básicos dejando de lado la implementación de las herramientas TIC. 
Por otro lado, Sumba et al. (2020) señalan que las tècnicas de aprendizaje son innovadoras por la forma de adaptarse a diversos recursos tecnològicos por lo que esto es un desafio o un nuevo reto para los docentes ya que no estan aconstumbrandos a implementar ese tipo de recursos en sus metodos de enseñanza por lo que deben capacitar a los estudiantes,tener una comunicación con cada uno de ellos,relacionar el aprendizaje con experiencias del mundo real para motivarlos a poder usar los recursos tecnologicos.El recurso màs importante es la comunicación afectiva entre docente y alumno la cual debe ser agradable dando confianza a cada uno de ellos utilizando para ello las herramientas digitales que permitan comunicarse de forma sincrónica o asincrónica. Por medio de los estudios en los resultados señalan que el $98.8 \%$ de los docentes señalan tener un gran dominio sobre este conocimiento. En lo referente al conocimiento tecnológico pedagógico el $99.2 \%$ de los docentes refieren poseer y aplicar este conocimiento. Esto eso debe en gran parte a los docentes ya que debido a la nueva normalidad de educación realizan la educación mediante recursos tecnológicos como las computadoras, tablets, laptops y una conexión a internet. Por lo que las TIC cobran una gran relevancia dentro del proceso de enseñanza y aprendizaje, convirtiéndose en la plataforma base para el desarrollo de la educación en tiempos de distanciamiento y confinamiento social.

Por otro lado, Carrillo y Toca (2019) señalan que una de las ventajas de la tecnologia digital fue el desarrollo de las capacidades de cada estudiante en los procesos de enseñanza y aprendizaje como sus aplicaciones demandan mayor difusión y socialización, particularmente entre los docentes de los países en desarrollo. Se recalcó que tuvo un alto porcentaje en la enseñanza remota desde casa con el apoyo de los padres de familia y los docentes que innovaron estrategias para que los estudiantes puedan aprender mediante las diversas plataformas virtuales sin ninguna complicación.

Y finalmente Vialart (2020) destacó que las diferentes tecnologías virtuales proporcionan recursos para desarrollar procesos de aprendizaje. En resumen, se implementó en el modelo de guía virtual en función de la construcción del conocimiento de los estudiantes, para el desarrollo del pensamiento, a través del aprendizaje a medida que los procesos sociales cooperan y practican la nueva enseñanza. En la educación, la virtualización actúa como una extensión de la clase a distancia, pero principalmente apoyada por la comunicación en curso entre docente y alumno. En una de sus conclusiones, lo que indica la educación remota o virtual debido a los factores de pandemia, la virtualización debe 
considerarse la posibilidad de acortar la distancia, enriquecer el proceso de aprendizaje de la educación y el desarrollo de los capítulos de las lecciones normales sin tener que suspender las clases ya que sin la suspensión,los materiales o las actividades pueden acceder a todo el tiempo y beneficiar a cada uno de ellos.

\section{CONCLUSIÓN O CONSIDERACIONES FINALES}

Como primera conclusión, se obtuvo que debido a la coyuntura actual es indispensable que el sector educación implemente el uso de la tecnología, para que tanto los estudiantes como los docentes puedan seguir desarrollando sus actividades como normalmente lo hacían antes de la llegada del COVID-19.

Como segunda conclusión, el Estado peruano ha venido facilitando los medios para que diversas escuelas, instituciones y universidades puedan sufrir la transformación digital que tanto requieren.

Como última conclusión, las estrategias que emplea el gobierno no están dando resultados positivos, ya que la población no se encuentra satisfecha, por ende, el incluir todos los factores posibles para encontrar una estrategia adecuada en beneficio de la transformación digital en el ámbito de la educación sigue siendo parte de un gran reto.

\section{LISTA DE REFERENCIAS}

Aguilar Gordón, F. (2020). From face-to-face learning to virtual learning in pandemic times. Estudios Pedagógicos, 46(3), 213-223. doi:http://dx.doi.org/10.4067/S0718-07052020000300213

Anaya Figeroa, T., Montalvo Castro, J., Calderon, A., \& Arispe Albureque , C. (2021). Escuelas Rurales en el Perù:Factores que acentuan las brechas digitales en tiempos de pandemia y recomendaciones para para reducirlas. Educación, 30(58). doi:http://dx.doi.org/10.18800/educacion.202101.001

Argüelles Cruz, A., García Peñalvo, F., \& Ramírez Montoya, M. (2021). Education in Latin America: Toward the Digital Transformation in Universities. Radical Solutions for Digital Transformation in Latin American Universities, 93-108. doi:https://doi.org/10.1007/978-981-16-3941-8_6

Carrillo Rodríguez, J., \& Toca Torres, E. (2019). Los entornos de aprendizaje inmersivo y la enseñanza a ciber-generaciones. Educao e Pesquisia, 45. doi: https://doi.org/10.1590/S1678-4634201945187369 
Cayo Rojas, C., \& Agramonte Rosell, R. (2020). Desafíos de la educación virtual en Odontología en tiempos de pandemia COVID-19. Revista Cubana de Estomatología, $57(3)$.

http://scielo.sld.cu/scielo.php?script=sci_arttext\&pid=S003475072020000300017

Connor Chick, R., Travis Clifton, G., Peace , K., Propper, B., Hale, D., Alseidi, A., \& Vreeland, T. (2020). Using Technology to Maintain the Education of Residents During the COVID-19 Pandemic. Journal of Surgical Education, 77(4), 729-732. doi:https://doi.org/10.1016/j.jsurg.2020.03.018

Cueva Gaibor, D. (2020). La tecnología educativa en tiempos de crisis. Conrado, 16(74), 341-348. http://scielo.sld.cu/pdf/rc/v16n74/1990-8644-rc-16-74-341.pdf

Gajardo Espinoza, K., \& Díez Gutiérrez, E. (2021). Evaluación educativa durante la crisis por COVID-19: una revisión sistemática urgente. Estudios pedagógicos (Valdivia), 47(2). doi:http://dx.doi.org/10.4067/S0718-07052021000200319

García Leal, M., Medrano RodríguezI, H., Vázquez Acevedo, J., Romero Rojas, J., \& Berrún Castañón, L. (2021). Experiencias docentes del uso de la tecnología educativa en el marco de la pandemia por COVID-19. Revista Informaciòn Cientifica, 100(2). http://scielo.sld.cu/pdf/ric/v100n2/1028-9933-ric-100-02e3436.pdf

Guedes dos Santos, J., Lorenzini Erdmann, A., Schlindwein Meirelles, B., Marcellino de Melo Lanzoni, G., Pecini da Cunha, V., \& Ratchneewan , R. (2017). Integrating Quantitative and Qualitative data in mixed methods research. Texto \& Contexto Enfermagem, 26(3). doi:https://doi.org/10.1590/0104-07072017001590016

Gómez Arteta, I., \& Escobar Mamani, F. (2021). Virtual Education in times of pandemic:Increasing Social Inequality in Perù. Scielo Prepints. doi:https://doi.org/10.1590/SciELOPreprints.1996

Huanca Arohuanca, J., Supo Condori, F., Sucari Leon, R., \& Supo Quispe, L. (2020). El problema social de la educación virtual universitaria en tiempos de pandemia, Perú. Revista Innovaciones Educativas, 22(1). doi:http://dx.doi.org/10.22458/ie.v22iespecial.3218

Iivari , N., Sharma , S., \& LeenaVentä , O. (2020). Digital transformation of everyday life - How COVID-19 pandemic transformed the basic education of the young 
generation and why information management research should care? International Journal of Information 55. doi:https://doi.org/10.1016/j.ijinfomgt.2020.102183

Levano Francia, L., Sanchez Diaz , S., Guillén Aparicio, P., Herrera Paico, N., \& Collantes Inga, Z. (2019). Competencias digitales y educación. Propósitos y Representaciones, 7(2). doi:http://dx.doi.org/10.20511/pyr2019.v7n2.329

Núñez Moscoso, J. (2017). Mixed methods in education research: towards a reflexive use. Cadernos de Pesquisa, 47(164). doi:https://doi.org/10.1590/198053143763

Ordorika, I. (2020). Pandemia y educación superior. Revista de la educación superior, 49(194).http://www.scielo.org.mx/scielo.php?script=sci_arttext\&pid=S018527602020000200001

Sànchez Flores, F. (2019). Fundamentos epistémicos de la investigación cualitativa y cuantitativa: Consensos y disensos. Revista Digital de Investigación en Docencia Universitaria, 13(1), 102-122. doi:http://dx.doi.org/10.19083/ridu.2019.644

Sumba Nacipucha, N., Cueva Estrada, J., Conde Lorenzo, E., \& Mármol Castillo, M. (2020). Enseñanza superior en el Ecuador en tiempos de COVID 19 en el marco del modelo TPACK. Revista San Gregorio(43). doi:https://doi.org/http:10.36097/rsan.v1i43.1524

Tobi, H., \& Kampen, J. (2018). Research design: the methodology for interdisciplinary research framework. Quality \& Quantity, 52, 1209-1225. doi:https://doi.org/10.1007/s11135-017-0513-8

Vialart Vidal, M. (2020). Estrategias didácticas para la virtualización del proceso enseñanza aprendizaje en tiempos de COVID-19. Educación Médica Superior, 34(3).

http://scielo.sld.cu/scielo.php?pid=S0864-

$21412020000300015 \&$ script=sci_arttext\&tlng=en 